\title{
Las representaciones sociales del cáncer y de la quimioterapia en la familia del paciente oncológico
}

\section{Social Representations of Cancer and Chemotherapy in the Cancer Patient's Family \\ As representações sociais do cáncer e da quimioterapia na família do paciente oncológico}

\author{
Ximena Palacios-Espinosa*, María Isabel González* \\ Universidad del Rosario, Bogotá, Colombia \\ Bruna Zani* \\ Universidad de Bolonia, Italia
}

Doi: dx.doi.org/10.12804/ap133.03.2015.09

\section{Resumen}

Esta investigación cualitativa tuvo como objetivo develar las representaciones sociales (RS) del cáncer y la quimioterapia en 25 familiares de pacientes oncológicos adultos con quienes se realizaron entrevistas en profundidad y asociaciones libres. Se utilizó el programa Atlas.ti para el análisis de los datos, con base en la teoría de las RS. Se encontró que el familiar del paciente cumple un papel protagónico; tiene funciones diversas como el acompañamiento y la contención y está tan implicado como el paciente en el proceso de enfermedad. Así, los familiares representan el cáncer y la quimioterapia de manera predominantemente negativa, lo que favorece la presencia de ansiedad y la percepción de sufrimiento.

Palabras clave: teoría de las representaciones sociales; representaciones sociales; cáncer; quimioterapia; familia. fbstract

This qualitative study aimed to analyze the Social Representations (SR) of cancer and chemotherapy in 25 families of adult cancer patients with whom in-depth interviews were conducted and who participated in a free association test. We used Atlas.ti for the data analysis which were analyzed was done based on SR theory. It was found that the patient's family member plays a central role; they have various functions such as support and containment are as involved as the patient in the disease process. Thus, the family members represent cancer and chemotherapy in a predominantly negative way, favoring the presence of anxiety and suffering. Keywords: social representation theory; social representations; cancer; chemotherapy; family.

* Ximena Palacios-Espinosa y María Isabel González, Programa de Psicología, Universidad del Rosario; Bruna Zani, Scuola di Psicologia e Scienze della Formazione.

La correspondencia relacionada con este artículo debe ser enviada a Ximena Palacios Espinosa. Correo electrónico: ximena.palacios@urosario.edu.co

Para citar este artículo: Palacios-Espinosa, X., González, M. I. \& Zani, B. (2015). Las representaciones sociales del cáncer y de la quimioterapia en la familia del paciente oncológico. Avances en Psicología Latinoamericana, 33(3), 497-515. doi: dx.doi. org/10.12804/apl33.03.2015.09 


\section{Resumo}

Esta pesquisa qualitativa teve como objetivo revelar as Representações Sociais (RS) do cáncer e a quimioterapia em 25 familiares de pacientes oncológicos adultos com quem se realizaram entrevistas em profundidade e associações livres. Utilizou-se Atlas ti para a análise dos dados que se analisaram com base na Teoria das RS. Encontrou-se que o familiar do paciente joga um papel principal; tem funções diversas como o acompanhamento e a contenção, e está tão implicado quanto o o paciente no processo de doença. Assim, os familiares representam ao cáncer e à quimioterapia de forma predominantemente negativa, favorecendo a presencia de ansiedade e a percepção de sofrimento.

Palavras-chave: teoria das representações sociais; representações sociais; cáncer; quimioterapia; família.

La familia del paciente oncológico constituye un grupo de actores siempre presente en el escenario de la oncología. Se trata de personas cuyo sufrimiento es tan intenso como el del paciente; así lo demuestra el estudio de Hinds (1992), el cual indica que la familia del paciente con cáncer sufre porque teme a la soledad, a la incertidumbre frente al futuro del paciente y al suyo propio, a las alteraciones en su estilo de vida, a las dificultades en la comunicación, a la ausencia de apoyo y a la desesperanza y adicionalmente, como lo expresa De Feudis (2003), porque asimilar informaciones médicas complejas y tomar decisiones sobre el tipo de tratamiento constituyen un gran estrés para los familiares de una persona a quien se le ha diagnosticado un cáncer. También otros estudios han confirmado el impacto del cáncer en las esferas afectiva, emocional, cognitiva y comportamental en el paciente con cáncer y en su familia (Giammona \& Malek, 2002; Libert et al., 2006). No obstante, a pesar de que con el diagnóstico del cáncer se cierne, entre otras, una amenaza de disgregación relacional, las necesidades de la familia suelen verse desplazadas por las del paciente, porque de ellos se espera que desempeñen el rol de cuidador informal, que sean la red de apoyo del paciente y que estén incondicionalmente con él.

Los estudios sobre familia de pacientes oncológicos se han centrado en diversos factores, como el estrés de su cuidado (Chaturvedi, 2012), la depresión (Kim, Carver, Rocha-Lima \& Shaffer, 2013), las estrategias educativas (Chiquelho et al., 2011), el temor a la recurrencia (Vivar, Whyte \& Mcqueen, 2010); pero no en el significado que tiene para ellos la enfermedad y su tratamiento convencional, lo que podría ser, entre otros, mantenedor de sus temores, del sufrimiento y del impacto negativo de la experiencia.

Precisamente, este estudio tiene el objetivo de develar los significados del cáncer y de la quimioterapia para la familia del paciente oncológico. Dado este propósito, se eligió como marco de referencia teórico la teoría de las representaciones sociales (TRS), pues favorece el estudio de las relaciones entre fenómenos sociales y cognoscitivos, comunicación y pensamiento. Las representaciones sociales (RS) permiten crear información, por cuanto su función es familiarizarnos con lo extraño, de acuerdo con las categorías de la cultura en la que estamos inmersos o a la que pertenecemos (Moscovici, 1988).

Los elementos constitutivos de las RS se explican a partir de dos procesos fundamentales que las forman y estructuran: objetivación y anclaje, que a su vez son responsables de su emergencia y organización. La objetivación es un proceso a través del cual los grupos sociales construyen un saber común basado en intercambios y opiniones compartidas. En cuanto al anclaje, es un proceso mediante el cual se trata de enraizar la representación en el espacio social para darle uso en la cotidianidad. Permite comprender cómo se construye una RS en relación con los valores, las creencias y los conocimientos de los que dispone el grupo social de donde ella ha emergido; en consecuencia, se integra a una red ya existente de significados. Es decir, el anclaje supone una integración cognoscitiva, caracteriza- 
da por la versatilidad, un proceso que tiene cuatro modalidades según el planteamiento de Jodelet: (a) asignación de sentido; (b) instrumentalización del conocimiento (adaptación del conocimiento que se ha adquirido de manera reciente con las necesidades sociales de expresión o de comprensión de la realidad vivida, que le da al individuo cierta sensación de seguridad al percibir algún grado de control sobre el ambiente y de reconocer su lugar en él); (c) integración de los procesos: anclaje y objetivación, y (d) enraizamiento en el sistema de pensamiento (conocimiento previo e información reciente se unen simultáneamente en el pensamiento, originando un nuevo contenido) (Valencia, 2007). Entonces, la objetivación permite que la información se transforme en una imagen-representación, mientras que el anclaje indica cómo se utiliza y modula esta en beneficio de los grupos (Valencia, 2007).

Una RS siempre se insertará en un marco de referencia preexistente, bien sea en forma de conversión (alterando el marco de pensamiento de origen) o de adaptación (insertándose en un sistema ya conocido) y se caracterizará por su dinamismo individual y sociocultural, sobre todo en referencia al lenguaje y al uso de la información. Además, en la formación de las RS, la objetivación y el anclaje transforman las ideas pasadas en un contenido mental concreto. Así, las ideas no familiares pueden ser familiares al vincularse con episodios familiares históricos o culturalmente familiares (Joffe, 2002).

Desde el punto de vista epistemológico, Banchs (2000) propone dos aproximaciones para el análisis de las RS: estructural (relacionada con la psicología social cognitiva de Norteamérica con representantes como Abric y su teoría del núcleo central) y procesual (emparentada con el interaccionismo simbólico procesual y con representantes como Jodelet y Doise). Este estudio cualitativo está enmarcado en la aproximación procesual, pues, como lo propone Banchs (2000), interesan los procesos sociocognoscitivos de procesamien- to de la información y la funcionalidad de las RS en la creación y en el mantenimiento de prácticas sociales. Desde esta aproximación, el ser humano es productor de sentidos; pueden analizarse las producciones simbólicas, los significados y el lenguaje (Banchs, 2000).

Aunque mucho menos que para el cáncer, algunos investigadores se han interesado en develar las RS de la quimioterapia (Cannone et al., 2004; Regnier-Denois, Poirson, Soum-Pouyalet \& Chauvin, 2009). No obstante, la población de estudio no suele ser la familia, sino el paciente y el personal de salud encargado de atenderlo.

\section{Objetivos}

Además de develar las RS sobre el cáncer y la quimioterapia en familiares de pacientes oncológicos adultos, son objetivos de este estudio explorar el conocimiento, así como describir el campo de representación y las actitudes del familiar sobre estos.

\section{Metodología}

\section{Tipo de estudio, población y muestra}

Este es un estudio cualitativo, enmarcado dentro de la aproximación procesual de las RS, que permite aproximarse y explorar los significados del cáncer y de la quimioterapia en los familiares de pacientes oncológicos adultos. De hecho, participaron once familiares mujeres, seleccionadas por conveniencia que fueron entrevistadas sobre su experiencia con el cáncer y con la quimioterapia. Sus edades oscilan entre los 18 y 41 años (45.4\%) y entre los 41 y los 60 años (45.4\%); casadas o en unión libre $(81.8 \%)$ o viudas $(18.1 \%)$. La mayoría nació fuera de Bogotá (72.7\%) tanto en zona rural como en la urbana; pero varias de ellas $(63.3 \%)$ viven en Bogotá ${ }^{1}$ en el momento de la entrevista. Todas viven en estratos socioeconómicos bajos

1 Ciudad capital de Colombia. 
(1 a 3), aunque predomina el estrato $2(63.6 \%) \mathrm{y}$ los bajos niveles educativos. Todas tienen hijos, se declaran católicas $(90.9 \%)$ practicantes $(81.8 \%)$ y la mayoría son amas de casa (45.4\%). Sus familiares tienen diferentes tipos de cáncer en estadios avanzados, para lo cual reciben quimioterapia intravenosa y predomina el parentesco con ellos de madre, esposa e hija.

Estos once familiares y otros catorce (para un total de veinticinco) realizaron asociaciones libres alrededor de las palabras: cáncer y quimioterapia. Diecinueve mujeres y seis hombres. Predominó la participación de las hijas (28\%) y la esposa (20\%).

\section{Contexto cultural, geográfico y económico de los participantes}

Colombia, país ubicado en Suramérica, tiene una capital (Bogotá) y 32 departamentos, clasificados en seis estratos socioeconómicos que en orden ascendente van del bajo-bajo (1) al alto (6), ${ }^{2}$ tanto en la zona urbana como en la rural (Consejo Nacional de Política Económica y Social, 2005). En general, en los hogares pobres (estratos 1 a 3), que son a los que pertenecen el $100 \%$ de los familiares de pacientes oncológicos que participaron en el estudio, las necesidades básicas se encuentran insatisfechas en alguna medida (33\% en el estrato $1 ; 12 \%$ en el $2 ; 4 \%$ en el 3 ) (Consejo Nacional de Política Económica y Social, 2005). Según el Censo General de 2005 (Departamento Administrativo Nacional de Estadística, 2005), en el Departamento de Cundinamarca (donde se encuentra la capital) predominan los bajos niveles educativos. Lo anterior permite evidenciar la vulnerabilidad en la que se encuentra esta población. Más de la mitad de los pacientes oncológicos en Colombia son diagnosticados en etapas avanzadas del cáncer (Instituto Nacional de Cancerología, 2012), por lo que la mortalidad e incapacidad son elevadas, y las necesidades de cuidado, indispensables. Esto implica alta dedicación de los familiares a este menester. Adicionalmente, si bien en Colombia la afiliación al Sistema General de Seguridad Social en Salud da derecho al cubrimiento del tratamiento con radioterapia, quimioterapia o trasplante de médula ósea para el cáncer, ${ }^{3}$ la tramitología es intensa y los familiares son quienes suelen asumir este tipo de procesos.

\section{Técnicas y procedimiento para la recolección de la información}

Al considerar la escasez de los antecedentes empíricos sobre las RS del cáncer y de la quimioterapia en los familiares del paciente oncológico, se seleccionaron métodos cualitativos para la recolección y el análisis de los datos: entrevistas en profundidad y asociaciones libres, que permitieron develar el significado del objeto de representación para esta población. Estas dos fuentes de datos se combinaron a través de un proceso de triangulación; al respecto es necesario indicar que los participantes comparten características similares que hacen viable tal proceso.

Un psicólogo experto en psiconcología entrevistó a los familiares utilizando un protocolo de once preguntas, de las cuales se aplicaron las siguientes con el propósito principal de promover la narrativa de los entrevistados sobre el cáncer y la quimioterapia: (a) hábleme acerca de cuando supo que su familiar estaba enfermo de cáncer; (b) ¿qué es para usted el cáncer?; (c) ¿cómo ha sido la experiencia de vivir esta enfermedad?; (d) ¿cómo ha afectado su vida esta experiencia?; (e) ¿qué es importante en su vida ahora?; (f) ¿qué cosas le han ayudado a afrontar esta experiencia?; (g) ¿ha habido algún momento durante su experiencia con el cáncer en el cual haya sentido que no podía afrontarlo?; (h) ¿cómo ha sido la experiencia de que su familiar

2 Ley 142 de 1994; Ley 286 de 1996; Ley 505 de 1999; Ley 689 de 2001; Ley 732 de 2002; Ley 812 de 2003; Ley 921 de 2004.

3 Resolución 5261 de 1994. 
reciba quimioterapia? Cuénteme desde la primera vez que se la administraron. Adicionalmente, se preguntaron algunos datos sociodemográficos como: la edad, el estado civil, el parentesco con el paciente, el lugar de origen, el lugar de residencia, el estrato socioeconómico, el número de hijos, la ocupación actual, la religión, el tipo de cáncer del familiar, el estadio del cáncer y si el familiar estaba recibiendo o no quimioterapia.

Las asociaciones libres las realizaron de manera individual tanto los once familiares que fueron entrevistados, como otros catorce (para un total de veinticinco), que se encontraban en la sala de espera de quimioterapia y consulta externa o en el consultorio o las salas de espera de hospitalización, si su familiar se encontraba hospitalizado. Esta técnica se eligió por ser de fácil comprensión, sencilla de administrar y adaptable (De Rosa, 2002) a los intereses del presente estudio. A cada uno de los participantes se le entregaron dos hojas. En una estaba escrita la palabra cáncer, y en la otra, la palabra quimioterapia. En cada hoja estaba descrita la instrucción que, además, fue proporcionada por el investigador principal, indicando que debajo de cada palabra debían escribir todas las palabras en las que pensaran cuando veían la palabra cáncer/quimioterapia. Se indicó que idealmente fueran mínimo cinco para cada palabra. Una vez escritas todas las palabras, debían ponerle en frente un signo + si el sentimiento experimentado frente a esta palabra era positivo, un signo - si el sentimiento era negativo y un signo $=$ si el sentimiento era de indiferencia. No obstante, se hizo hincapié en la importancia de intentar asignar un sentimiento $+\mathrm{o}-$, más que de indiferencia.

Tal y como lo propone De Rosa (2002), con las asociaciones libres se pueden obtener medidas globales de los componentes actitudinales de las RS si se calculan los índices de polaridad y de neu- tralidad. El primero, "permite obtener una medida sintética de la evaluación y la actitud implícita en el campo representacional, mientras que el segundo es una medida de control (si se asume que una polaridad altamente positiva corresponde con una falta de neutralidad y vice-versa)" (De Rosa, 2002, p. 185).

Se realizaron entrevistas y asociaciones libres hasta saturar la información.

Con previa autorización del participante, se grabaron en audio todas las entrevistas y luego se transcribieron, con el propósito de favorecer la riqueza y la fidelidad de los datos. Inicialmente, se realizó un preanálisis de una entrevista y de un grupo focal, del que emergieron unas primeras categorías. En un segundo momento se analizaron todas las entrevistas, de las cuales emergieron las categorías que se definieron como definitivas y, simultáneamente, se inició la interpretación de los datos con la codificación y la elaboración de memos. Todo lo anterior con el uso del software Atlas.ti versión 5.0. Finalmente, estos se organizaron y dieron lugar a la escritura de una propuesta comprensiva de las RS del cáncer y de la quimioterapia entre los familiares de los pacientes oncológicos. Así mismo, se utilizó la teoría fundamentada de Strauss y Corbin como método de análisis, ya que permite revisar y comparar de manera continua los datos recolectados para así elaborar una teoría de la realidad (Bautista, 2011). "Es una forma de pensar acerca de los datos y de poderlos conceptualizar" (Bautista, 2011, p. 126) en el marco teórico de referencia de esta investigación, la TRS. Con base en esta última se realizó la interpretación de los resultados.

Las asociaciones libres también se categorizaron, agrupando las frases o palabras asociadas en cada categoría e indicando su frecuencia a través del programa Excel; así mismo, se obtuvieron los índices de polaridad ${ }^{4} \mathrm{y}$ de neutrali-

4 Índice de polaridad $(\mathrm{P})=$ Número de palabras positivas - Número de palabras negativas/Número total de palabras asociadas "El rango del índice está entre -1 y +1 . Si P está entre -1 y - .05 (el valor puede recodificarse como 1 o como -1 ), entonces la mayoría de palabras tendrán una connotación negativa. Si P está entre $-.04 \mathrm{y}+.04$ (el valor puede recodificarse como 2 o como 0 ), entonces las palabras tanto positivas como negativas tienden a ser iguales. Y si P está entre $+.04 \mathrm{y}+1$ (el valor puede recodificarse como 3 o como +1), entonces la mayoría de las palabras tienen una connotación positiva” (De Rosa, 2002, p. 185). 
dad, ${ }^{5}$ según los criterios propuestos por De Rosa (2002). El análisis de estas asociaciones contribuyó de manera específica a (a) describir el campo de representación construido por los familiares de los pacientes sobre el cáncer y la quimioterapia, y (b) identificar el componente evaluativo y actitudinal de la representación social sobre estos dos objetos.

\section{Aspectos éticos}

El estudio, clasificado como de riesgo mínimo según la Resolución 008430 de 1993, recibió aprobación del Comité de Ética de la Clínica en la cual recibían atención los familiares (pacientes oncológicos) de los participantes, de quienes se obtuvo el consentimiento informado verbal; todos conocieron el estudio porque presenciaron la toma del consentimiento a sus familiares (pacientes oncológicos) cuando fueron entrevistados o sus familiares fueron quienes les comentaron que habían participado en el estudio y los remitieron a participar en este. El investigador siempre les indicó el objetivo de la investigación y los aspectos relacionados con la confidencialidad, el respeto del anonimato y la participación voluntaria.

\section{Resultados}

Las categorías emergentes del análisis permitieron establecer una guía para la interpretación de los datos que se presenta a continuación. En la narrativa se van desarrollando y van apareciendo cada una de ellas.

Si bien se presentan algunos subtítulos (información, actitud y afrontamiento), los resultados se presentan redactados en forma de una cadena lógica comprensiva con la que se pretende ilustrar la compleja experiencia del familiar frente a la experiencia del cáncer y del tratamiento oncológico de su pariente, lo que da lugar a la elaboración de un esquema comprensivo (figura 1). En consecuencia, los subtítulos no saturan la información sobre un tema, sino que la enuncian. Esta se agota en el desarrollo del discurso que se ha construido a partir de la triangulación y del análisis interpretativo de los datos.

\section{Representaciones sociales del cáncer}

Conocimiento. El familiar del paciente oncológico cumple un papel protagónico en la experiencia del cáncer y de la quimioterapia. Se trata de personas que, por lo general, pertenecen al núcleo familiar del paciente: los padres, los hermanos, la pareja o los hijos. Por ejemplo, en las asociaciones libres realizadas $(N=25)$, es evidente que el $24 \%$ estuvo representado por hombres cuidadores (padre, $8 \%$; hijo, $8 \%$, y esposo, $8 \%$ ); mientras que el $76 \%$ restante por mujeres cuidadoras (hija, 28\%; esposa, 20\%; hermana, 16\%, y madre, $12 \%$ ).

Sin embargo, todos los familiares implicados en el cuidado del paciente oncológico tienen funciones que se desarrollan en un continuo que va desde el acompañamiento hasta la verdadera contención en el afrontamiento de la enfermedad; continuo en el que el familiar está tan implicado afectiva y emocionalmente como el paciente. Lo anterior significa que el familiar del paciente no es un simple espectador de la experiencia que vive su ser querido; es un actor y, por tanto, su experiencia frente al cáncer es tan profunda y compleja como la del paciente: sufre; experimenta sensaciones de malestar emocional intenso, con percepción de pérdida de control, de impotencia que se produce tanto por la enfermedad de su pariente y el tratamiento

5 Índice de neutralidad $(N)=[$ Número de palabras neutras - (número de palabras positivas + número de palabras negativas) $] /$ Número total de palabras asociadas

"El rango del índice está entre $-1 \mathrm{y}+1$. Si $N$ está entre -1 y -.05 pocas palabras tienen una connotación neutral (=baja neutralidad). Si $N$ está entre -.04 y +.04 , las palabras neutrales tienden a ser iguales a la suma de las palabras positivas y negativas. Si $N$ está entre +.04 y +1 , muchas palabras tienen una connotación neutral (=alta neutralidad)" (De Rosa, 2002, p. 186). 


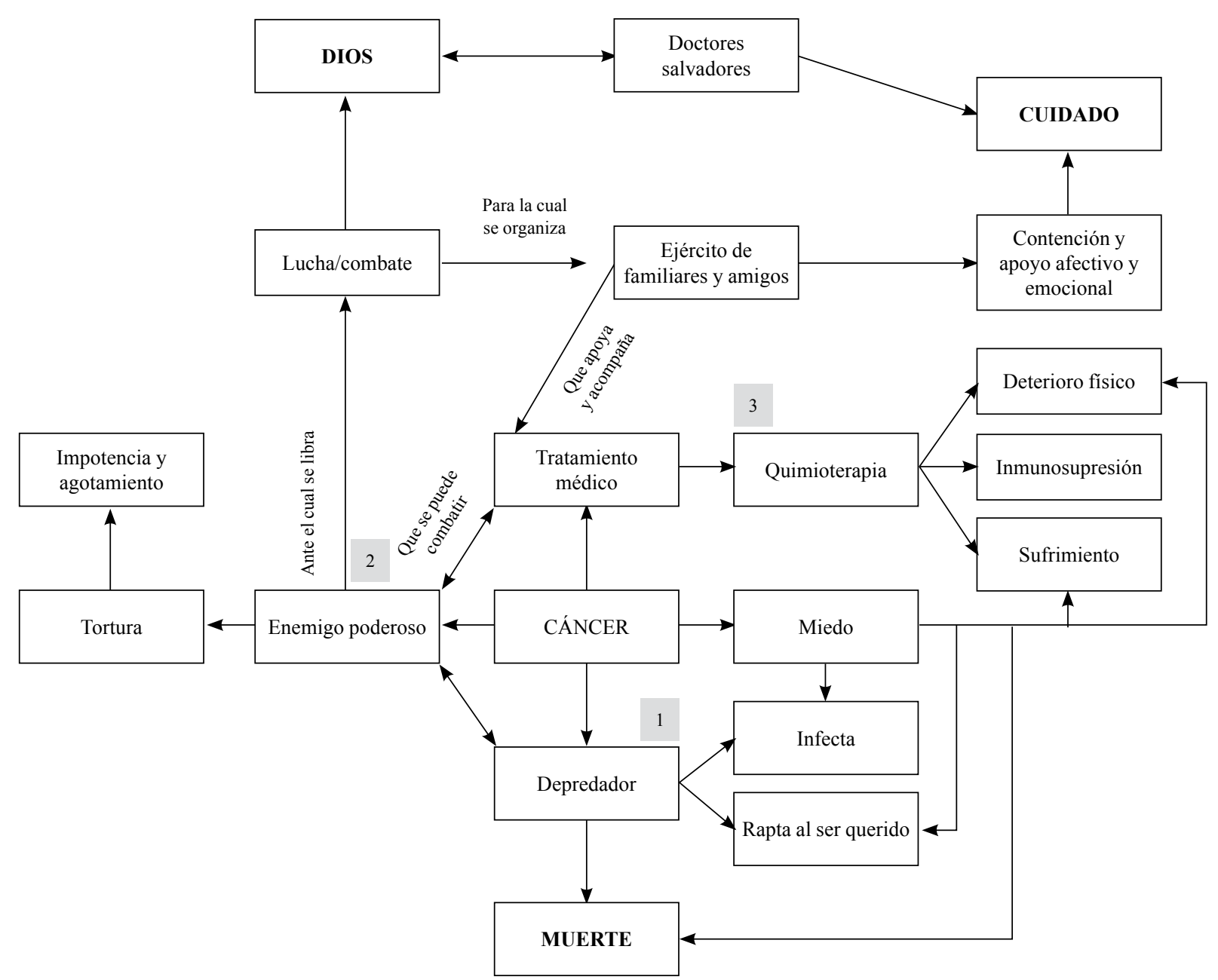

Figura 1. Esquema comprensivo del campo representacional del cáncer y de la quimioterapia para la familia del paciente oncológico adulto

que este recibe como por los eventos implicados en la vivencia de la enfermedad: el impacto del diagnóstico, la toma de decisiones frente a las opciones terapéuticas, la vida, la muerte, el fracaso terapéutico, etc.

De hecho, se considera que el cáncer es una enfermedad familiar no solo porque exista en ella un componente hereditario claramente identificado, sino por las implicaciones que simbólicamente tiene en la familia. De las asociaciones libres realizadas por los familiares, una de las categorías emergentes fue justamente familia y los sentimientos y emociones asociados a ella son ambivalentes: algunos positivos elicitados por ideas como la unión, la unión familiar y el apoyo, y otros negativos elicitados por ideas como trabajo para la familia, distanciamiento de los hijos, dejar al hijo solo y estrés en la familia (tabla 1).

En general, los conocimientos sobre el cáncer que provienen - entre otros - de diversas fuentes indican que el cáncer es:

1. Una enfermedad mortal o impredecible: “... yo eso sí lo tengo muy claro; el cáncer no se cura; yo eso sí lo sé porque el hematólogo habló con nosotros; el cáncer no tiene cura, entonces, de pronto que lo ayude a controlar sí pero no a curar..." $(2: 124)$. 
Tabla 1

Asociaciones libres realizadas por los familiares de los pacientes oncológicos frente a la palabra estímulo cáncer e índices de polaridad y de neutralidad

\begin{tabular}{|c|c|c|c|c|}
\hline \multirow{2}{*}{ Categoría } & \multirow{2}{*}{ Frecuencia } & \multicolumn{3}{|c|}{ Sentimiento/emoción asociados } \\
\hline & & Positivo & Negativo & Neutro \\
\hline Muerte & 32 & 0 & 32 & 0 \\
\hline Dolor y sufrimiento & 27 & 0 & 27 & 0 \\
\hline Familia & 8 & 4 & 4 & 0 \\
\hline Cáncer incapacitante & 6 & 0 & 6 & 0 \\
\hline Enfermedad grave & 10 & 0 & 10 & 0 \\
\hline Afrontamiento & 6 & 1 & 5 & 0 \\
\hline Esperanza & 4 & 4 & 0 & 0 \\
\hline Miedo y amenaza & 15 & 0 & 15 & 0 \\
\hline Síntomas y consecuencias del cáncer & 9 & 0 & 9 & 0 \\
\hline Tristeza y depresión & 10 & 0 & 10 & 0 \\
\hline Cáncer implacable & 5 & 0 & 5 & 0 \\
\hline Otros & 1 & 0 & 1 & 0 \\
\hline Total: & 133 & 9 & 124 & 0 \\
\hline Porcentaje: & 100 & 6.8 & 93.2 & 0 \\
\hline Índice de polaridad $(\mathrm{P})$ : & -0.86 & (tendencia a & ación nega & \\
\hline Índice de neutralidad $(N)$ : & -1 & (baja neutral & & \\
\hline
\end{tabular}

2. Con nombres específicos, que deteriora a la persona que lo padece: "Sí. A ver, $N$ tiene un linfoma no Hodgkin donde es cutáneo pero de igual manera están comprometidos todos sus ganglios y todo su sistema inmunológico y como tal, está propenso a una enfermedad en sus riñones, sus pulmones, tiene todas sus proteínas perdidas porque un medicamento se las quitó y como tal, la enfermedad de él, estoy bien informada" (1:32).

3. Lo que dicen en la televisión, en la radio, en los libros, en la internet, los comentarios de la gente o la información que les han otorgado los miembros del equipo de salud acerca de lo que es el cáncer, lo que produce y los tratamientos que le hacen a la gente para manejarlo: "Pues lo que me ha explicado el médico, lo que he es- cuchado de otras partes y más también he leído sobre el cáncer" (3:35).

4. Una enfermedad que requiere tratamientos médicos especializados como cirugía, quimioterapia, radioterapia, de exámenes y procedimientos especializados: "Pues también aprendí, porque cuando uno entra a este proceso no sabe nada... nada. Pero también sé que hay las más fuertes (quimioterapias), y de mayor intensidad y su intención es destruir células malas pero también destruye las células buenas" (1:34).

5. El producto de la exposición al riesgo: "Ya luego, cuando yo me quedaba en la sala yo sí empecé a leer y vi que cuando a uno le dan esas pastillas de la sustitución hormonal puede producir cáncer" (10:20). 
6. Una enfermedad temida, especialmente grave: "Yo, cómo le dijera, como una enfermedad muy mala... Creo que es una enfermedad muy pero muy grave, algo malo que crece en uno y que es delicado" (11:14).

7. Una enfermedad capaz de raptar a su ser querido y de llevarlo sin compasión, un monstruo implacable: “... porque es que esa enfermedad se encarga de eso... de comerse al cuerpo por dentro como viviendo dentro de la persona y acabándolos. O por lo menos uno aquí también escucha eso así de otra gente que uno va conociendo y cuando yo le cuento a otras personas que mi mami tiene eso pues toda la gente le dice a uno que tenaz, que pobrecita, que eso no le dejemos hacer todas esas cosas que nos ofrecen porque la van a matar" (9:27).

8. Un ser personificado en un tumor que incapacita y que devora sin clemencia la vida de su familiar: "Por ejemplo, el doctor F le dijo a mi hermana que no fuera a tomar Ensure ni vitaminas, porque al alimentarme pues también está alimentando al tumor" (7:59).

9. Un ser cuyo apellido es MUERTE: "Pues es que con solo la palabra cáncer es muerte..." (2:38).

Actitud. El cáncer se representa entonces como un personaje vivo con características casi humanas; se transforma, se camufla, es implacable, voraz, un intruso capaz de destruir la vida de una persona. En especial, genera un intenso sufrimiento, lo que genera una actitud negativa hacia esta experiencia vital:

Mejor dicho... esto es lo peor; es algo que uno no se puede imaginar sino hasta que lo vive. (LLORA). Usted no imagina y no se lo deseo a nadie lo que es ver a la mamá sufriendo, verla calladita sudando del dolor y a veces quejándose por la noche. Eso yo paro oreja cuando me acuesto y apenas la oigo quejarse y yo quedo parada otra vez. Ella dice que no, que me acueste, pero yo sé que sufre y yo también sufro por ella y por mi viejito que lo veo sufrir y yo no puedo sino ver por mi mami porque está muy malita y casi no tiene alientos ni nada. Cuando ella está aquí hospitalizada, por lo menos, yo puedo ver un poquito de mi papá y de mis hijitas; pero si está en la casa, eso es imposible, porque ella solamente quiere estar conmigo. (9:27)

En el núcleo familiar se ha construido un significado de la enfermedad; se ha aprendido su valor y sus consecuencias (tabla 1). Se sabe que esta enfermedad genera dificultades, costos, hospitalizaciones, que distancia de la tierra (es decir, del lugar de procedencia), que genera toda clase de síntomas físicos (como desaliento, hemorragias, somnolencia) y está asociado con la quimioterapia. Todo ello genera una actitud negativa de parte del familiar hacia el cáncer. Así lo confirman los índices de polaridad calculados y la baja neutralidad que se evidenció frente a esta palabra estímulo (tabla 1).

Afrontamiento. Así que desde el momento en que la familia se entera del diagnóstico de cáncer, inicia el despliegue de esfuerzos por combatir a ese ser que les anuncia la muerte de su pariente. De forma prácticamente inmediata, se hará evidente esa red de apoyo familiar cuya compleja dinámica se alimenta de apoyar al paciente y, a la vez, de recibir apoyo entre los diferentes miembros que la componen. Esto se traduce en:

1. La presencia de la familia durante el proceso de enfermedad, desde la sospecha del diagnóstico hasta la remisión o la muerte: "No pues a toda la familia, estuvo mi familia y pues la familia de él estuvieron todos muy pendientes" (4:8).

2. Unión: "Además con esto, la familia se unió más, lo llaman todos los días, están pendientes ahora de todo. La niña pequeña está con mi mamá, pero igual ellas están pendientes de la niña, de lo que se le ofrezca, le llevan la leche, que las compotas, que el Nestum, el abuelo también está muy pendiente de ellas, de la mayorcita. Para qué, pero se han unido más" (5:51). 
3. Compañía, contención y fortaleza, reconocimiento de que el amor familiar es fundamental para poder afrontar el proceso de enfermedad y de tratamiento: "... hemos tratado de que tenga una vida muy armoniosa, para que ella esté bien con todo el apoyo familiar (LLORA)" (6:17).

4. Valor del respaldo, del afecto incondicional, de la contención afectiva, de los consejos, de la solidaridad: "Cuando ella se cortó el cabello yo iba a ir a cortármelo con ella pero me dijo que no, que le comprara unos gorritos y así fue, todo eso lo hemos hecho" (6:18); de la colaboración: "No importa, entre toda la familia juntamos el dinero y se lo dimos" (7:10).

5. Todos los familiares hacen sus aportes de acuerdo con sus posibilidades y conocimientos.
Igualmente, hacen presencia otras personas que constituyen la red de apoyo social con la cual esta familia va a interactuar durante el proceso de enfermedad. Entrarán y saldrán de ella diversos actores, pero siempre se tejerán nuevas relaciones y se fortalecerán otras que al final favorecerán la contención afectiva y emocional del paciente: "Y nosotras nos hicimos muy amigas; pero hoy a las dos se nos fueron las personas que amábamos tanto. A ella su esposo y a mí, mi hijo" (12:42).

Representaciones sociales de la quimioterapia. El familiar teme profundamente las consecuencias del cáncer en su ser querido; lo ven como generador de miedo y amenaza (tabla 2) y, a la vez, teme las consecuencias de la quimioterapia, las cuales se representan bajo la figura de dolor físico,

Tabla 2

Asociaciones libres realizadas por los familiares de los pacientes oncológicos frente a la palabra estímulo quimioterapia e índices de polaridad y de neutralidad

\begin{tabular}{|c|c|c|c|c|}
\hline \multirow{2}{*}{ Categoría } & \multirow{2}{*}{ Frecuencia } & \multicolumn{3}{|c|}{ Sentimiento/emoción asociados } \\
\hline & & Positivo & Negativo & Neutro \\
\hline Dolor físico y sufrimiento & 10 & 0 & 10 & 0 \\
\hline Náuseas y vómito & 9 & 0 & 9 & 0 \\
\hline Alopecia & 6 & 0 & 6 & 0 \\
\hline Medicamentos químicos & 15 & 9 & 6 & 0 \\
\hline Miedo y amenaza & 3 & 0 & 3 & 0 \\
\hline Muerte & 5 & 0 & 5 & 0 \\
\hline Otros síntomas & 16 & 0 & 16 & 0 \\
\hline Esperanza/optimismo & 15 & 15 & 0 & 0 \\
\hline Curativa & 9 & 9 & 0 & 0 \\
\hline Propiedades de la quimioterapia & 18 & 5 & 13 & 0 \\
\hline Ambiente hospitalario & 4 & 0 & 4 & 0 \\
\hline Otros & 4 & 2 & 2 & 0 \\
\hline Total: & 114 & 40 & 74 & 0 \\
\hline Porcentaje: & 100.0 & 35.1 & 64.9 & 0 \\
\hline Índice de polaridad $(\mathrm{P})$ : & -0.30 & Las palabras posit & s y negativa & a ser iguales \\
\hline Índice de neutralidad $(N)$ : & -1 & Baja neutralidad & & \\
\hline
\end{tabular}


sufrimiento, náuseas, vómito, alopecia, muerte y otros síntomas, donde predomina una actitud poco neutral en la que la ambivalencia actitudinal es evidente hacia este tratamiento (tabla 2). Así lo confirmaron los índices de polaridad y de neutralidad calculados para estas asociaciones (tabla 2) que denotan, pese a la tendencia a que las palabras positivas y negativas sean iguales, un mayor componente actitudinal negativo: "Y había que hacerle los baños y toda esa cosa y nos dimos cuenta que eso no era calidad de vida. Yo pienso que eso es restarle a la persona lo que le queda para vivir porque no aporta nada" (10:22). "Sí, pasó de ser algo que no conocía a ser algo malo, algo que no recomendaría y que no me haría. Y si volviera a pasar, yo no lo volvería a hacer" (10:27).

El familiar teme lo que el cáncer y la quimioterapia logren en ese cuerpo que empieza a ver frágil. Para él son evidentes los cambios corporales físicos/fisiológicos (como pérdida de peso, modificaciones en el color o en el aspecto de la piel, dolor, debilidad, alopecia, náuseas, vómito, aspecto frágil), o funcionales (como pérdida del apetito, incapacidad para movilizarse solo). El familiar experimenta también, simbólicamente como si fuese sobre su propio cuerpo, el impacto de los cambios corporales, la evidencia de su deterioro: "Es que la quimioterapia son venenos, hacerla hace sufrir, duele, angustia... es como estar muerto en vida" (10:25). Y aunque quisiera escuchar a ese cuerpo, entrar en un episodio delirante que se lo permitiera, sabe que él solo lo ve y que es su ser querido el que lo siente: "Igual fue muy duro que a ella se le cayera el cabello, que se le cayeran las cejas; una ventaja es que mi mamá no ha sido tan vanidosa ni usaba maquillaje ni estaba pendiente del cabello ni del peinado; pero igual fue terrible" (6:18).

La quimioterapia se convierte en una amenaza, así como el dolor que desencadena los más intensos sentimientos de impotencia y que nutre, entre otras muchas experiencias, los sentimientos de tristeza y la depresión al ver que al ser amado le duele y no poder eliminar su dolor. Se siente angustia, com- pasión, un sufrimiento intenso y una confirmación más de la fragilidad del paciente:

Y al otro día cuando yo le conté a H, él se sonrió y nunca más le volvió a doler. Entonces yo me volví íntima amiga de la Virgen. Pero íntima. Yo pienso que lo más duro del cáncer es eso, el dolor. Porque ¿qué puedes hacer ante eso? Ante el vómito le das galletas, lo acuestas, pero ante el dolor, no hay nada que puedes hacer. (10:73)

No obstante, este familiar es capaz de comprender que la quimioterapia es la forma médica disponible para controlar o calmar el cáncer:

Pienso yo que es para ayudar a controlar la enfermedad, mas no para curarla. (2:65); [y que es inherente al diagnóstico]: Hum... sí, es lo que nos dijo el Dr. X que mi mamá tiene que hacerse cuando le confirmó que tenía el cáncer en la matriz. Y pues eso es lo que se le ha hecho a mi mamá y pues es muy complicada. Para mí la quimioterapia es un tratamiento que es el que hay que hacer para el cáncer pero muy bravo... duro. (11:17)

El familiar del paciente oncológico comprende que los efectos de la quimioterapia son tan intensos y peligrosos que con frecuencia implicarán el ingreso al hospital. El hospital... ese escenario que se convierte durante el proceso del cáncer en un hogar alterno... un hogar sin amor distinto al que pueda profesarle al paciente un familiar que desplaza otras obligaciones inherentes a su existencia, para acompañarlo, para cuidarlo. Un hogar en el que hay que aprender que sus características son los chuzones, los medicamentos, las náuseas, la soledad, la indiferencia de muchos de los miembros del equipo de salud, las restricciones, los horarios, los trámites. El hospitalizado, el paciente, lo vive, lo siente y tiene un papel protagónico en ese escenario hospitalario. En cambio, el familiar se siente invisible ¿O lo es?; es el extra... el que sirve para hacer el trámite ante el sistema de salud, el que 
hace las filas, el que recibe una mala noticia tras otra, el que deja su casa, su trabajo, su vida para cuidar de su ser querido.

Pese a todo lo que lucha con la tramitología, eventualmente el familiar del paciente tiene una actitud positiva hacia el sistema de salud; paradójicamente, agradece a lo que tiene derecho y minimiza los malestares vividos en esa experiencia:

Gracias a Dios también que el seguro le ha dado a mi mamita todo porque si no... imagínese. Yo digo que eso de Colsubsidio es una gran cosa, doctora, porque yo he visto en las facturas eso es carisísimo, doctora; uno cómo podría pagar eso. Dígame usted. Entonces yo sí estoy muy agradecida con el seguro para qué (9: 32$)$.

Sin embargo, otros familiares reniegan de la atención recibida por parte del sistema y en consecuencia, su actitud es negativa:

Sí, lo de la salud es terrible; ver la lucha para los medicamentos, para que le den su tratamiento, por lo menos en el caso de $\mathrm{N}$, que es un guerrero $\mathrm{y}$ que va para un trasplante, que ni siquiera estamos en un listado pero no en una lista que tenga el gobierno, se puede decir que entre las enfermedades raras y llegar uno a puro pulso, discutiendo con el uno, discutiendo con el otro, para hacerle entender al estado que está primero la vida de él. (1:17)

Pero ese familiar, percibe que a nadie le interesan sus necesidades, no hay con quién hablar de ello porque la familia espera que el cuidador sea el que contiene emocionalmente y en el ambiente hospitalario, el familiar no ve al psicólogo, solo ve un grupo de "doctores" en el que solo le queda la opción de confiar. Solo, asustado y lleno de angustia, se enfrenta con resignación, con calma y aprende a tener paciencia y a comprender que está ahí porque su ser querido lo necesita. En un afán por ajustarse y sobrevivir a todo el impacto recibido, decide considerar que el apoyo afectivo y emocional está en la misma familia, en sí mismo o en Dios: "El mejor psicólogo digo yo doctora es uno mismo y Dios; uno tiene que poner de su parte y eso es lo que yo le digo a mi hermana y a mi hijo; hay que ayudarse y ella ahora también se lo dice a él" (7:72).

El familiar, como el paciente, termina sumergido en un mundo nuevo, el del cáncer, en el que debe aprender a asumir el rol de cuidador del paciente:

Pues, a ver, mirándolo por un lado positivo, ha sido un cambio grandísimo en mi vida porque yo era dependiente, autodependiente, demasiado. Pedía permiso hasta para ir a la esquina, entonces muy dependiente. Esto me llevó a tomar autonomía propia, a ser fuerte. Yo lloraba por una novela, entonces aprendí a ser fuerte ante mi propia novela. Han sido cosas positivas. En cuanto a lo negativo, los momentos en que uno se siente impotente, en que siente que no puede hacer nada bueno. Entre lo negativo, es eso, el sentirse impotente ante el dolor... Pero bueno, se sacan fuerzas... entonces va como enganchado lo positivo con lo negativo. (1:39)

Aunque sin entrenamiento (pero también sin reparos), el familiar se vuelca sobre las necesidades del paciente, y a tientas o con el insumo que le ha dado la experiencia de la vida, se dedica a cuidarlo:

Pero no, pues nosotros hemos tratado de ser muy consecuentes con ella, de tenerla muy cuidada, de decirle bueno: "Estamos en tratamiento, vamos a hacer esto, vamos a hacer aquello", porque la idea es que ella esté con nosotros, es prepararla para la cirugía, nosotros ya sabemos que lo que se nos viene no es fácil, pero así mismo la vamos respaldando, la vamos motivando, porque de nada nos sirve que nosotros le demos todo pero si ella no tiene ese deseo de hacer las cosas, pues realmente no se va a lograr. $(6: 15)$.

Afrontamiento. Para ello, el familiar hace uso de las estrategias de afrontamiento que le pertene- 
cen y desarrolla otras que le ayudarán a sortear o a sufrir más los problemas que se le presentarán durante el proceso:

1. Luchar activamente contra la enfermedad pero en el marco del realismo:

Entonces, con él es con quien llevamos el proceso porque es que su mente es lo que ayuda. Es el gran ejemplo de agradecerle todos los días, con decirle chica, que allá todos tratamos de llevar una vida normal, todos tienen que ir a estudiar, a su trabajo y seguir la vida normalmente, todo alrededor de él pero él mismo hace que todo sea normal. Primero que todo Dios y luego su mente [...] Pero yo digo que primero, Dios, segundo, la mentalidad de él, tercero su familia. En cuanto a medicamentos que uno le puede haber hecho, pues lo que le han inyectado porque después de esa experiencia tan dura, ya vino como el duelo, lo fuimos superando y aquí estamos frente a la realidad. (1:10)

2. Estar calmado y ser paciente: “... y él me dijo que no me pusiera así, que él iba a salir de esto, que al contrario, que le diera apoyo. Entonces desde ahí yo me hice la promesa de que no iba a volver a llorar delante de él ni nada, ya me calmé..." (2:11).

3. Animar al paciente y ser optimista:

... él se quedó: ¿cómo? Y cuando llegamos a la casa me dijo: "Yo no sabía que la cosa fuera tan grave"; es que él inclusive en la cita que tuvimos con la psicóloga antes del trasplante, él le decía a la psicóloga que él no se veía mal, o sea que él no se veía mal, pero nosotros sí lo veíamos mal, pero pues tampoco se lo estábamos diciendo, nosotros tratábamos era de estar con él, darle ese ánimo... (2:11)

\section{Evitar:}

Yo me quedo en la casa y organizo el apartamento, soy muy adicta al oficio, me gusta tener todo limpio y A peleaba conmigo por eso, porque no puedo ver que, mejor dicho, entonces me pongo, arreglo mi apartamento, volteo las cosas, eso me ayuda mucho o me ayuda salir al centro comercial y comprarme siquiera un helado; ya con eso yo me compongo. Entonces si no hago nada en la casa y ya tengo todo hecho, digo voy a ir al centro comercial me compro un helado, me siento un rato y ya descargo y vuelvo a mi casa otra vez, ya normal. $(2: 55)$

5. Recurrir a ayudas de tipo religioso. Pero, además de estas estrategias, los miembros de la familia disponen de una serie de insumos adquiridos en el proceso de conformación y consolidación como grupo familiar y de su desarrollo individual: creencias religiosas y populares, tradición y espiritualidad. Las creencias populares son todas aquellas construcciones e interpretaciones que ha elaborado el familiar alrededor del cáncer, de la quimioterapia, de las razones por las que hay que realizarla o de hacer otro tipo de manejo por ejemplo basados en remedios caseros y por qué estos son efectivos; son construcciones que se alimentan de la tradición oral, de la historia personal, de los comentarios, que mantienen la ejecución de prácticas populares y de actitudes positivas o negativas hacia la enfermedad y el tratamiento:

... tratamos de que tenga muy bien sus defensas porque si ella tiene bien sus defensas, va a estar bien, va a resistir la quimio; es lo más importante para que no se nos descompense. Y pues, igual, si la van a preparar para una cirugía es más fácil y pues igual tratar de darle lo más posible. A nosotros nos pasaba con mi papá que se le bajaban las defensas, se le bajaba el potasio y entonces se nos caía y se ponía muy mal; entonces si nosotros la tenemos bien de defensas, por lo menos ella se va a poder mover por sí misma y tolerar la quimio para que no la afecte tanto. (6:17) 
Las creencias religiosas incluyen la realización de prácticas religiosas, rituales y la firme convicción en el poder y la palabra de Dios: "También yo ahí le he pedido mucho a mi Dios que le de fuerzas y me dé también a mí para que yo la sepa sobrellevar" (5:72). De hecho, esta religiosidad suele estar estrechamente ligada a la fe; pero no necesariamente a la espiritualidad. La fe implica confiar en alguien, en que aquello que se desea que suceda, reconocer la evidencia de los indicios que Dios envía para sobrellevar la enfermedad y el tratamiento con mayor resignación y tranquilidad. Es una convicción fundamentada en creencias mágico-religiosas. La convicción en que uno puede controlar o tener algún grado de control en lo que viene relacionado con la enfermedad. Es un paliativo que permite tener la ilusión de que el sufrimiento no será tan intenso: “Además, todo depende de la fe que uno le ponga a esto. De hecho yo se lo digo a mi mamá, que le ponga fe que se tome las cosas y haga las cosas con confianza que ella va a estar bien. Y si no le sirven, pues por lo menos no le hacen daño" (6:68).

La espiritualidad es el legado de vida que el familiar recibe del paciente: "Nosotras afortunadas con ese ejemplo de vida que él nos enseña todos los días" (1:26); el sentido de la enfermedad, de la muerte y de la vida, la misión que asume en su vida al cuidar al paciente con cáncer:

Entonces me dijo que yo tenía que vivir mi vida. Y yo le dije: “¿Qué es vivir mi vida? ¿Volverme a casar?’. A mí no me interesa volverme a casar. Entonces me dijo algo que yo nunca he recordado exactamente pero es algo como que en este momento siento que me vas a dejar de querer, no sé decirte qué me dijo. Y yo le respondí a eso que me dijo: "Hace mucho tiempo yo te dije una cosa en la salud, pero incondicionalmente en la enfermedad (LLORA), en la alegría, pero como nunca en la tristeza (LLORA), en la riqueza pero siempre en la pobreza hasta que la muerte nos separe, no más allá de la muerte porque mi amor del alma se va contigo (LLORA) Ay ¡no! Es que esto no es fácil”. (10:11)

$\mathrm{Y}$ son justamente todos los insumos mencionados hasta el momento aquellos que actuarán como moduladores del apoyo y del cuidado que le proporcionan al paciente. El diagnóstico de cáncer alerta al familiar del paciente oncológico, pero el anuncio de que será operado, irradiado o que recibirá quimioterapia, así como los evidentes síntomas y consecuencias del cáncer, detonan la puesta en marcha de todas las estrategias conocidas por él o por personas en quienes siente alguna credibilidad (como pueden ser amigos, conocidos, familiares de otros pacientes oncológicos, etc.), para atacar al cáncer y todos los efectos producidos por el tratamiento.

Dedicarse al cuidado de un ser querido puede implicar "cuidar al que antes me cuidaba", comprender que los roles cambian, que la enfermedad es de todos y para todos; implica también hacerse cargo de los requisitos que impone el Sistema General de Seguridad Social para lograr la atención del enfermo y supone intentar establecer una relación con el personal de salud que garantice que su ser querido recibirá un trato digno en todo momento. Este familiar siente y asume esta responsabilidad y debe enfrentarse a relaciones tensionantes que generan distancia y malestar con los miembros del equipo de salud: "Después de que llegamos aquí a que el oncólogo nos diga qué pensaba respecto a los exámenes que traía, que honestamente los hicieron acá mismo es, entonces nos dicen que no tiene sino dos meses de vida" (1:10); o a aquellas basadas en el buen trato, la cordialidad, el respeto pero no en la confianza necesaria para poder comunicar las necesidades:

Aquí todo ha sido excelente. Yo digo que esta clínica sí fue una bendición de Dios, porque aquí a mi mamita la han tratado muy bien, la han atendido mucho y las niñas aquí son muy formales y el doctor $\mathrm{x}$, no, ese sí que es un tesoro, un amor. Yo digo 
que gracias a mi Dios que el doctor $\mathrm{x}$ fue el que le correspondió a mi mamita, porque él le da ánimos y le dice: "tranquila, doña $\mathrm{C}$, que todo va a estar bien... que vamos para adelante, que lo importante es que no tenga dolor”. (9:31).

No, señora. Yo no le he dicho ni mi mami tampoco porque ellos no creen en eso y sí de pronto se ponen es bien bravos. (9:89)

O una relación centrada en el médico y en la que el paciente legitima que el trato recibido no sea el deseado, bien sea por temor a las reacciones del médico, a que la atención médica se vea afectada negativamente, porque el médico está muy ocupado o porque él tiene el derecho de decir las malas noticias aunque no sea de una manera adecuada:

No, señora; es que yo no veo que eso sea útil, porque igual nosotros estamos haciendo lo que hay que hacer y no estamos faltando. Lo único que yo le doy es natural, inorgánico, o sea que yo no veo que haya necesidad de pronto de que él se moleste o haya problemas. $(5: 58)$

O de forma eventual, el familiar se verá enfrentado a una relación centrada en la cordialidad, en la confianza y en la posibilidad de establecer una comunicación efectiva con los miembros del equipo de salud:

Buenísima, ya le digo, es la persona que nos levanta la moral, es la persona que nosotros lo vemos como un Dios a él. Claro, primero mi Señor, luego el doctor F. Mire, yo salí de este hospital, primero mi hermana, luego mi hijo, la forma como nos trata $\mathrm{y}$ todo. No he conocido una persona tan especial como ese doctor. (7:36)

Cualquiera que sea la relación que se establezca, es el resultado de una lotería que se juega el familiar. Por lo general, se ha aprendido que específicamente el médico es un ser poderoso, capaz de dar o de devolver la salud e incluso la vida, un salvador: "Y pues también el doctor X pues él es muy bueno; mi mamá lo adora a ese doctor y dice que gracias a él es que ella está acá en este mundo todavía" (11:97). Por ello es en quien se deposita la confianza en la curación de su ser querido, independiente del trato que profese: "Además tenemos un súper médico, un poco bravito, pero es el mejor hematólogo que hay y hace sus cosas bien" (5:53).

\section{Discusión y conclusiones}

Los resultados de esta investigación ponen en evidencia que la información que tienen los familiares del paciente oncológico acerca del cáncer y de la quimioterapia proviene de la experiencia actual, de lo que han escuchado en los medios de comunicación y de otras personas que han tenido conocidos o parientes con cáncer. Esta información es una construcción social, cultural, refleja la historia de la persona y su realidad. Les permite significar la experiencia de recibir un diagnóstico que alarma a la gente. La información científica sobre el cáncer y sobre la quimioterapia ha permeado la cultura popular, mezclándose de manera inevitable con el sentido común. Es entonces imposible considerarlos opuestos o incompatibles, por lo que, en acuerdo con Bargenter (1995), habría que pensar el sentido común como parte de un universo consensual que entre otras cosas incluye al conocimiento científico.

Para la familia del paciente oncológico, el cáncer es un depredador capaz de infectar y de raptar al ser querido; ese depredador es la representación de la muerte. De hecho, estas son dos representaciones que han trascendido los tiempos, como lo reportó Salcedo (2008), al indicar que históricamente el cáncer se ha asociado con la muerte y la putrefacción, otorgando a esta enfermedad la capacidad de consumir y devorar el cuerpo. Sin duda, para cualquier persona, pero en especial para la familia del paciente con cáncer, la mencionada 
representación asusta. Se teme al deterioro físico y al sufrimiento. El cáncer es una condena a muerte, tal y como lo han reportado Rees, Fry, Cull y Sutton (2004); Insa, Monleón y Espallargas (2010), y Marie, Dany, Cannone, Dudoit y Duffaud (2010), un enemigo poderoso que tortura y genera impotencia y agotamiento, pero también el responsable de que se decida librar una lucha, una batalla, respaldados en el poder de Dios que es quien ilumina a los doctores, actúa a través de ellos, para cuidar al paciente. Esto es el reflejo de lo que Herzlick (1973) describió como la enfermedad representada socialmente como una ocupación, lo que significa que el paciente, en este caso el familiar, se involucra en el proceso de curación o en el proceso de afrontamiento adaptativo de la enfermedad crónica. De acuerdo con Ehrenzweig-Sánchez (2007), se le teme al dolor, a las metástasis, a la inminencia de la muerte y a los efectos secundarios de los tratamientos oncológicos. En general, los familiares centran su discurso alrededor del sufrimiento y de la muerte como lo plantea Murray (2002).

El presente estudio es una aproximación a la comprensión de cuanto viven los familiares de un paciente oncológico adulto; pero en especial, sus necesidades, sus temores, más allá de centrarse en el estrés de estos o en la necesidad de entrenamientos para optimizar su afrontamiento. En consecuencia, este estudio pone en evidencia que el equipo de salud, que ha reconocido que el cáncer es una enfermedad familiar por razones previamente enunciadas, debe, sin excepción, ocuparse de la familia del paciente oncológico, de tener en su equipo psiconcólogos capaces de dar apoyo emocional y comprender el significado que tiene para un familiar cuidar, pero también confrontarse con la muerte, con el cáncer y con la quimioterapia de su ser querido. Así mismo, este estudio permite comprender que el estrés del familiar no solamente está ligado al cuidado y a las largas horas de vigilia en las que las demandas son inagotables, sino que sus propias RS son una fuente de estrés agudo y de franco malestar emocional.
De acuerdo con lo anterior, este estudio puede proporcionar elementos a los diferentes actores de los ambientes hospitalarios (médicos, enfermeros, trabajadores sociales, psicólogos, etc.) que interactúan con la familia de los pacientes con el ánimo de lograr una intervención, desde lo particular de su disciplina, que responda a sus necesidades afectivas y emocionales, que aborden y proporcionen soluciones a las dificultades en la relación médico-paciente-familia; que identifiquen factores de riesgo para la salud mental de los cuidadores; que permitan educar sobre el cáncer para promover así conductas de salud y prevenir la exposición al riesgo. En conclusión, los resultados de este estudio se constituyen en un insumo que permite aproximarse a la realidad de un grupo tan importante en el proceso del cáncer como el mismo paciente.

En lo referente a las limitaciones del estudio, hay que mencionar que es cualitativo, que apenas alcanza la descripción de un grupo poblacional pequeño. Por consiguiente, habrá que ampliar la investigación sobre las RS del cáncer y de la quimioterapia entre los familiares de pacientes oncológicos adultos (e incluso de niños) hasta encontrar una tendencia que permita el diseño de estudios mixtos que permitan realmente comprender, pero especialmente explicar, el afrontamiento del familiar del paciente oncológico en un sentido amplio. $\mathrm{Y}$, así, poder diseñar programas efectivos para el manejo de las necesidades psicológicas de este grupo poblacional.

\section{Referencias}

Banchs, M. A. (2000). Aproximaciones procesuales y estructurales al estudio de las representaciones sociales. Papers on Social Representations. Textes sur les Représentations Sociales, 9, 3.1-3.15.

Bargenter, A. (1995). Rethinking the relation between science and common sense: A comment on the current state of SR theory. Papers on 
Social Representations, 4, 1-78. Recuperado de: http://www.psych.lse.ac.uk/psr/PSR1995/4_ 1995Bange.pdf

Bautista, N. P. (2011). Proceso de la investigación cualitativa: epistemología, metodología y aplicaciones. Bogotá: Manual Moderno.

Cannone, P., Dany, L., Dudoit, E., Duffaud, F., Salas, S., \& Favre, R. (2004). Study of chemotherapy social representations: A way to analyze relations between patients and oncologists. Bulletin du Cancer, 91(3), 279-284.

Chaturvedi, S. K. (2012). Psychiatric oncology: Cancer in mind. Indian Journal of Psychiatry, 54(2), 111-118. doi: 10.4103/0019-5545.99529

Chiquelho, R., Neves, S., Mendes, Á., Relvas, A.P., \& Sousa, L. (2011). ProFamilies: A psycho-educational multi-family group intervention for cancer patients and their families. European Journal of Cancer Care, 20(3), 337-344. doi: 10.1111/j.1365-2354.2009.01154.x

Consejo Nacional de Política Económica y Social de la República de Colombia, Departamento Nacional de Planeación. (2005). Documento Conpes 3386. Plan de acción para la focalización de los subsidios para servicios públicos domiciliarios. Recuperado de: http://www.dane. gov.co/files/dig/CONPES_3386_oct2005_Focaliz_subsidios_servicios_publicos.pdf

Departamento Administrativo Nacional de Estadística. (2005). Boletín Censo general 2005. Departamento Cundinamarca. Recuperado de: http://www.dane.gov.co/files/censo2005/PERFIL PDF CG2005/25000T7T000.PDF

Giammona, A. J., \& Malek, D. M. (2002). The psychological effect of childhood cancer on families. Pediatrics Clinics of North America, 49(5), 1063-1081.

De Feudis, R. (2003). Il tempo dell'esitazione: la relazione di aiuto psicologico con la donna operata di cancro al seno. Terapia Familiare, 71, 49-68.

De Rosa, A. S. (2002). The "associative network": A technique for detecting structure, contents, po- larity and stereotyping indexes of the semantic fields. European Review of Applied Psychology, 52(3-4), 181-200.

Ehrenzweig-Sánchez, Y. (2007). Modelos de cognición social y adherencia terapéutica en pacientes con cáncer. Avances en Psicología Latinoamericana, 25(1), 7-21. Recuperado de dialnet.unirioja.es/servlet/fichero_articulo?codigo $=2542687 \&$ orden $=0$

Herzlick, C. (1973). Health and illness. London: Academic Press.

Hinds, C. (1992). Suffering: A relatively unexplored phenomenon among family caregivers of non-institutionalized patients with cancer. Journal of Advanced Nursing, 7(8), 918-925. doi: 10.1111/j.1365-2648.1992.tb02019.x

Insa, L. L., Monleón, M. A. B., \& Espallargas, A.P. (2010). El enfermo de cáncer: una aproximación a su representación social. Psicologia \& Sociedade, 22(2), 318-332. Recuperado de: http:// www.scielo.br/pdf/psoc/v22n2/12.pdf

Instituto Nacional de Cancerología. (2012). Anuario estadístico 2010. Bogotá: Buenos y Creativos. Recuperado de: http://www.cancer.gov.co/documentos/Anuario\%20Estaditico/2010/Anuario\%20Impreso_2010.pdf

Joffe, H. (2002). Social representations and health psychology. Social Science Information, 41(4), 559-580.

Kim, Y., Carver, C. S., Rocha-Lima, C., \& Shaffer, K. M. (2013). Depressive symptoms among caregivers of colorectal cancer patients during the first year since diagnosis: A longitudinal investigation. Psychooncology, 22(2), 362-367. doi: $10.1002 /$ pon. 2100

Ley 142 de 1994, por la cual se establece el régimen de los servicios públicos domiciliarios y se dictan otras disposiciones. Recuperado de http:// www.acueducto.com.co/wpsv61/wps/html/resources/empresa/LEY142DE1994.pdf

Ley 286 de 1996, por la cual se modifican parcialmente las Leyes 142 y 143 de 1994. Recuperado de http://camacol.co/sites/default/files/ 
base_datos_juridico/LEY_CONGRESO_NACION_0286_1996.pdf

Ley 505 de 1999, por medio de la cual se fijan términos y competencias para la realización, adopción y aplicación de la estratificación a que se refiere las Leyes 142 y 177 de 1994, 188 de 1995 y 383 de 1997 y los Decretos Presidenciales 1538 y 2034 de 1996. Recuperado de http://www.alcaldiabogota.gov.co/sisjur/normas/ Norma1.jsp? $i=185$

Ley 689 de 2001, por la cual se modifica parcialmente la Ley 142 de 1994. Recuperado de http://www. dane.gov.co/files/dig/Ley_689_2001.pdf

Ley 732 de 2002, por la cual se establecen nuevos plazos para realizar, adoptar y aplicar las estratificaciones socioeconómicas urbanas y rurales en el territorio nacional y se precisan los mecanismos de ejecución, control y atención de reclamos por el estrato asignado. Recuperado de http://www.alcaldiabogota.gov.co/sisjur/normas/Norma1.jsp?i=4736

Ley 812 de 2003, por la cual se aprueba el Plan Nacional de Desarrollo 2003-2006, hacia un Estado comunitario. Recuperado de http://www. alcaldiabogota.gov.co/sisjur/normas/Norma1. jsp? $\mathrm{i}=8795$

Ley 921 de 2004, por la cual se decreta el Presupuesto de rentas y recursos de capital y Ley de apropiaciones para la vigencia fiscal del 10 de enero al 31 de diciembre de 2005. Recuperado de http://www.secretariasenado.gov.co/senado/ basedoc/ley_0921_2004.html

Libert, Y., Merckaert, I., Étienne, A. M., et al. (2006). Les besoins psychosociaux et le soutien apporté aux patients attaints d'un cancer: une étude nationale belge. Oncology, 8(5), 465-476.

Marie, D., Dany, L., Cannone, P., Dudoit, E., \& Duffaud, F. (2010). Représentations sociales du cancer et de la chimiothérapie: enjeux pour la définition de la situation thérapeutique. Bulletin du Cancer, 97(5), 577-587. doi: 10.1684/ bed.2010.1036
Moscovici, S. (1988). The history and actuality of social representations. En U. Flick (Ed.), The psychology of the social (pp. 209-247). Cambridge: Cambridge University Press.

Murray, M. (2002). Connecting narrative and social representation theory in health research. Social Science Information, 41(4), 653-673. doi: 10.1177/0539018402041004008

Rees, G., Fry, A., Cull A., \& Sutton, S. (2004). Illness perceptions and distress in women at increased risk of breast cancer. Psychology \& Health, 19(6), 749-765. doi:10.1080/0887044041233 1279764

Regnier-Denois, V., Poirson, J., Soum-Pouyalet, F., \& Chauvin, F. (2009). La chimiothérapie par voie orale: représentations et pratiques des oncologues et des patients. Oral anti-cancer chemotherapy: social representations and medical practices. Psycho oncologie, 3(3), 168-175. doi: 10.1007/s11839-009-0140-2

Resolución 5261 de 1994, por la cual se establece el Manual de Actividades, Intervenciones y Procedimientos del Plan Obligatorio de Salud en el Sistema General de Seguridad Social en Salud. Resolución 8430 de 1993, por la cual se establecen las normas científicas, técnicas y administrativas para la investigación en salud. República de Colombia: Ministerio de Salud.

Salcedo, H. (2008). Representaciones sociales y metáforas del cáncer en los siglos XVII y XVIII: una antología de lugares comunes. Antípoda, 6 , 199-213. Recuperado de: http://dialnet.unirioja. es/servlet/articulo?codigo $=2777943$

Valencia, S. (2007). Elementos de la construcción, circulación y aplicación de las representaciones sociales. En T. Rodríguez \& M. L. García, Representaciones sociales: teoría e investigación. México: CUCSHUDG. Recuperado de: http://books.google. com.co/books?id=X3Eb7sKFaWMC\&p$\mathrm{g}=\mathrm{PA} 26 \& \mathrm{lpg}=\mathrm{PA} 26 \& \mathrm{dq}=D E+\mathrm{LA}+\mathrm{CON}-$ CIENCIA+COLECTIVA+A+LAS+REPRE- 
SENTACIONES\& source $=$ bl\&ots $=$ KCN6UbU-P6\&sig=oIBfptnCYtLf0y9kun4OPDcJ$\mathrm{A} 1 \mathrm{I} \& \mathrm{hl}=\mathrm{es} \# \mathrm{v}=$ onepage $\& \mathrm{q}=D E \% 20 \mathrm{LA} \% 20$ CONCIENCIA $\% 20$ COLECTIVA $\% 20 \mathrm{~A} \% 20$ LAS\%20REPRESENTACIONES\& $\mathrm{f}=$ fals
Vivar, C. G., Whyte, D. A., \& Mcqueen, A. (2010). 'Again': The impact of recurrence on survivors of cancer and family members. Journal of Clinical Nursing, 19(13/14): 2048-2056. doi: $10.1111 / \mathrm{j} .1365-2702.2009 .03145 . \mathrm{x}$

Fecha de recepción: 22 de agosto de 2014 Fecha de aceptación: 15 de abril de 2015 
